\title{
Quasinormal modes and their anomalous behavior for black holes in $f(R)$ gravity
}

\author{
Almendra Aragón ${ }^{1, \mathrm{a}}$, P. A. González ${ }^{1, \mathrm{~b}}$, Eleftherios Papantonopoulos ${ }^{2, \mathrm{c}}$, Yerko Vásquez ${ }^{3, \mathrm{~d}}$ \\ ${ }^{1}$ Facultad de Ingeniería y Ciencias, Universidad Diego Portales, Avenida Ejército Libertador 441, Casilla 298-V, Santiago, Chile \\ ${ }^{2}$ Physics Division, National Technical University of Athens, Zografou Campus, 15780 Athens, Greece \\ ${ }^{3}$ Departamento de Física y Astronomía, Facultad de Ciencias, Universidad de La Serena, Avenida Cisternas 1200, La Serena, Chile
}

Received: 9 December 2020 / Accepted: 23 April 2021 / Published online: 10 May 2021

(C) The Author(s) 2021

\begin{abstract}
We study the propagation of scalar fields in the background of an asymptotically de Sitter black hole solution in $f(R)$ gravity. The aim of this work is to analyze in modified theories of gravity the existence of an anomalous decay rate of the quasinormal modes (QNMs) of a massive scalar field which was recently reported in Schwarzschild black hole backgrounds, in which the longest-lived modes are the ones with higher angular number, for a scalar field mass smaller than a critical value, while that beyond this value the behavior is inverted. We study the QNMs for various overtone numbers and they depend on a parameter $\beta$ which appears in the metric and characterizes the $f(R)$ gravity. For small $\beta$, i.e. small deviations from the Schwarzschild-dS black hole the anomalous behavior in the QNMs is present for the photon sphere modes, and the critical value of the mass of the scalar field depends on the parameter $\beta$ while for large $\beta$, i.e. large deviations, the anomalous behavior and the critical mass does not appear. Also, the critical mass of the scalar field increases when the overtone number increases until the $f(R)$ gravity parameter $\beta$ approaches the near extremal limit at which the critical mass of the scalar field does not depend anymore on the overtone number. The imaginary part of the quasinormal frequencies is always negative leading to a stable propagation of the scalar fields in this background.
\end{abstract}

\section{Contents}

1 Introduction . . . . . . . . . . . . 1

$2 f(R)$ modified gravity ....................... 3

3 Scalar perturbations ............ 4

\footnotetext{
a e-mail: almendra.aragon@mail.udp.cl

be-mail: pablo.gonzalez@udp.cl

ce-mail:1papa@central.ntua.gr

de-mail: yvasquez@userena.cl (corresponding author)
}

3.1 The photon sphere family . . . . . . . . . 5

3.1.1 Photon sphere modes . . . . . . . . . . 5

3.1.2 Anomalous decay rate . . . . . . . 6

3.1.3 Analysis using the WKB method . . . . . 7

3.2 The de Sitter family . . . . . . . . . . . . 10

3.3 The dominance family . . . . . . . . . 11

4 Conclusions . . . . . . . . . . . . . 12

References . . . . . . . . . . . . . . . . . 14

\section{Introduction}

Modified theories of gravity in which the Einstein-Hilbert action is replaced with a generic form of $f(R)$ gravity have been introduced in an attempt to describe the early and late cosmological evolution. Another motivation to study such theories is the understanding of the existence of dark energy and dark matter consistent with the recent observations, without introducing new material ingredients that have not yet been detected by experiments [1-6]. Modifying the action not only affects the dynamics of the universe, it can also alter the dynamics at the galactic or solar system scales. Therefore, modified theories of gravity with curvature corrections provide a deeper understanding of General Relativity (GR).

Assuming that the gravitational Lagrangian is not only a linear function of $R$, variable modified theories of gravity were considered describing the cosmic evolution at early times, in which the gravitational Lagrangian contains some of the four possible second-order curvature invariants. Also models in which higher order curvature invariants as functions of the Ricci scalar were introduced in the gravitational Lagrangian, resulting in various $f(R)$ gravity models [7$16]$. Although such theories exclude contributions from any curvature invariants other than $R$, they could also avoid the Ostrogradski instability [17] which proves to be problematic for general higher derivative theories [18]. 
One of the first modifications of the Einstein Lagrangian density was proposed in [19]. A more natural modification of the Einstein Lagrangian is to add terms $R^{n}$, like the Starobinsky model $f(R)=R+\alpha R^{2}$ [20-22]. For $n<0$ such corrections become important in the late universe and can lead to self-accelerating vacuum solutions [23-26]. However, these models suffer from instabilities [27,28] and there are strong constraints from the solar system [29]. A wide range of phenomena can be explained by considering different $f(R)$ functions. Some discussions have been performed, such as on gravitational wave detection [30,31], early-time inflation [32], cosmological phases [33-35], the singularity problem [36], the stability of the solutions [37-39], and various other branches have been studied [40].

Spherically symmetric static solutions have been studied in $f(R)$ theories of gravity. In particular, it was shown that for a large class of models the Schwarzschild-de Sitter (SdS) metric is an exact solution of the field equations. However, in addition to the SdS metric, $f(R)$ theories typically also have new different solutions [41]. Also, static spherically symmetric perfect fluid solutions have been studied by matching the outside SdS-metric to the metric inside the mass distribution that leads to additional constraints that limit the allowed fluid configurations [42], while in [43] black hole solution were found with and without electric charge, and exact spherically symmetric solutions were discussed in [44-49]. Also, exact charged black hole solutions in $f(R)$ gravity theories with dynamic curvature in $D$-dimensions were discussed in [50].

A way to probe the behavior and the stability of black holes is the study of their quasinormal modes (QNMs) and quasinormal frequencies (QNFs) [51-55]. The QNMs depend on the black hole parameters and probe field parameters, and on the fundamental constants of the system and they are independent of the initial conditions of the perturbations. The QNMs are described by complex frequencies, $\omega=\omega_{R}+i \omega_{I}$, in which the real part $\omega_{R}$ determines the oscillation timescale of the modes, while the complex part $\omega_{I}$ determines their exponential decaying timescale (for a review on QNM modes see $[53,56])$.

The QNMs and QNFs have been calculated using various numerical and analytical techniques [57-62]. In the case of gravitational perturbations it was found that for the Schwarzschild and Kerr black hole background the longestlived modes are always the ones with lower angular number $\ell$. In the case of a massive probe scalar field it was found [63-66], at least for the overtone $n=0$, that, if the mass of the scalar field is small, then the longest-lived QNMs are those with a high angular number $\ell$, whereas if the mass of the scalar is large the longest-lived modes are those with a low angular number $\ell$. This inverted behavior appears when the mass of the scalar field exceeds a critical value, which corresponds to the value of the scalar field mass where the behavior of the decay rate of the QNMs is inverted and can be obtained from the condition $\operatorname{Im}(\omega)_{\ell}=\operatorname{Im}(\omega)_{\ell+1}$ in the eikonal limit, that is when $\ell \rightarrow \infty$. This anomalous decay rate for small mass scale of the scalar field was recently discussed in [67].

The study of the anomalous behavior of QNMs was extended to other asymptotic geometries, such as, Schwarzs -child-de Sitter and Schwarzschild-AdS black holes in [68]. It was found that the same behavior is present in the Schwarzschild-de Sitter background, i.e., the absolute values of the imaginary part of the QNFs decay when the angular harmonic numbers increase if the mass of the scalar field is smaller than the critical mass, and they grow when the angular harmonic numbers increase, if the mass of the scalar field is larger than the critical mass. Also it was found that the increase of the value of the cosmological constant results in the increase of the value of the critical mass of the scalar field. It was also found that the anomalous behavior is present in Schwarzschild-AdS black holes backgrounds; however, there is not a critical mass where the behavior in the decay rate is inverted. Also anomalous decay of QNMs in accelerating black holes was recently reported in [69], as well as for Reissner-Nordström black holes [70]. Furthermore, the anomalous behavior of QNMs was observed for fermionic field perturbations in Schwarzschild-de Sitter black holes [71].

Stability studies have been performed to $f(R)$ gravity theories. However, the stability analysis appears to be very difficult to be applicable to $f(R)$ gravity theories because it involves fourth-order derivative terms in the linearized equations [72]. One way to evade this problem is to transform $f(R)$ gravity into the corresponding scalar-tensor theory to remove the fourth-order derivative terms [73]. However, it is well known that a non-minimally coupled scalar makes the linearized field equations very intricate when compared to a minimally coupled scalar in the context of General Relativity [74]. To avoid this problem one may use conformal transformations to find the corresponding theory in the Einstein frame where a minimally coupled scalar field appears. The resulting theory can be considered as a scalar-tensor theory with a geometric (gravitational) scalar field. Then it was shown in $[75,76]$ that this geometric scalar field cannot dress a $f(R)$ black hole with hair, therefore, the no-hair theorem is respected in these models. Matter was introduced in [77] and a $R^{2}$ gravity model was discussed having a conformally coupled scalar field with associated Higgs-like potential and a static spherically symmetric black hole was found. However, the conformally coupled scalar field could not yield black hole hair, because it is regulated by the same parameters as appear in the metric. A scalar field minimally coupled to gravity with a self-interacting potential in a $f(R)$ theory was discussed in [78]. An exact hairy black hole solution was found similar to the BTZ black hole. Also the process 
of scalarization of $f(R)$ gravity theories was investigated in [79].

As we already discussed, knowledge of the QNMs and QNFs can give us vital information of the behavior, properties and the stability of black holes. In this work we will consider a specific $f(R)$ theory which accepts a black hole solution [80]. In this theory the Ricci scalar receives nonlinear correction terms which they introduce new parameters in the metric functions of the black hole solution, expressing the change of the curvature altering the strength of the gravitational force. In this theory we will consider a massless and a massive test scalar field scattered off the background black hole and we will calculate the QNMs and QNFs. The motivation of this work is to calculate the scalar field perturbations and study the behavior of QNMs and QNFs and see how they are effected by the presence of non-linear curvature terms which appear explicitly in the metric functions.

The manuscript is organized as follows: In Sect. 2 we give a brief review of the model considered for $f(R)$ gravity and its black hole solution. In Sect. 3, we study the scalar field stability and we calculate the QNFs of scalar perturbations numerically by using the pseudospectral Chebyshev method for each family of modes. In Sect. 3.1.3, we perform an analysis using the WKB method to get some analytical insight. Finally, our conclusions are in Sect. 4.

\section{$2 f(R)$ modified gravity}

We consider a generic action in $f(R)$ gravity depending on the Ricci scalar $R$ given by

$S=\frac{1}{2 k} \int d^{4} x \sqrt{-g} f(R)+S_{m}$,

where $S_{m}$ is the matter content of the theory. In [80] a specific ansatz for the function $f(R)$ was considered:

$f(R)=R+\Lambda+\frac{R+\Lambda}{R / R_{0}+2 / \alpha} \ln \frac{R+\Lambda}{R_{\mathrm{c}}}$,

where $\Lambda$ corresponds to the cosmological constant, $R_{\mathrm{c}}$ is a constant of integration and $R_{0}=6 \alpha^{2} / d^{2}$, with $\alpha$ and $d$ being free parameters. Then considering the spherically symmetric metric

$\mathrm{d} s^{2}=B(r) \mathrm{d} t^{2}-A(r) \mathrm{d} r^{2}-r^{2}\left(\mathrm{~d} \theta^{2}+\sin ^{2} \theta \mathrm{d} \phi^{2}\right)$,

where $A(r)=B(r)^{-1}$, a vacuum spherically symmetric solution was found in [80] with $B(r)=1-\frac{2 M}{r}+\beta r-\frac{\Lambda r^{2}}{3}$, where $\beta=\alpha / d$ is a real constant. For the range $R>>\Lambda$ and $R / R_{0}>>2 / \alpha$ the action reduces to

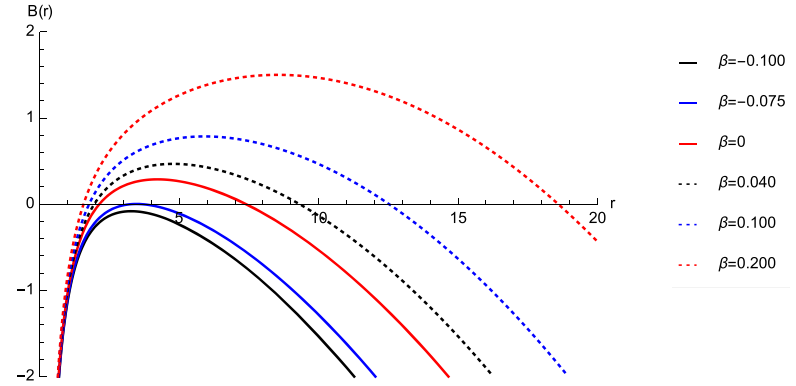

Fig. 1 The behavior of $B(r)$ as a function of $r$ for different values of the parameters $\beta$ with $M=1$ and $\Lambda=0.04$

$f(R)=R+R_{0} \ln \left(\frac{R}{R_{c}}\right)$,

and this is satisfied in the solar system range of $r<<d$. On the other hand, at large scales, for $\alpha<<1$ and $R \sim$ $R_{0} \sim \Lambda$, the action tends to $f(R) \sim R+\Lambda$ and agrees with cosmological observations. For the metric (3) the curvature scalar is given by

$R=\frac{6 \beta}{r}-4 \Lambda$,

and the action tends to a constant value asymptotically,

$f(R) \approx\left(-3 \Lambda-\frac{3 R_{0} \alpha \Lambda \ln \left(-3 \Lambda / R_{\mathrm{c}}\right)}{2 R_{0}-4 \alpha \Lambda}\right)+\mathcal{O}\left(r^{-1}\right)$.

It is worth to mention that the solution in both regimes was obtained as perturbation around the Einstein-Hilbert action and it was shown that within this approximation the solutions are consistent with the modified gravity equations. Also, the parameters of the theory were constrained by using the pioneer data, the flat rotation curve of galaxies and the cosmic microwave background radiation and Supernova Type Ia gold sample data giving $\beta \approx 10^{-26} \mathrm{~m}^{-1}, \beta d \approx 10^{-6}$ and $\beta d<<10^{-3}$, respectively [80]. However, the spacecraft's heat loss is a more plausible explanation for the anomaly than the cosmological one [81].

Our analysis considers a range of values of $\beta$, such that the metric represents a black hole solution with an event horizon and a cosmological horizon as it shown in Fig. 1. In this figure for a fixed value of the cosmological constant, we show the transition among black holes, near extremal $\left(r_{H} \approx r_{\Lambda}\right)$ and naked singularity, when the $\beta$ parameter goes from positive to negative values. Notice that, for $\beta=0$, the spacetime is described by a Schwarzschild-dS black hole. So, for $\beta$ positive (negative) the cosmological horizon radius is larger (smaller) than the Schwarzschild-de Sitter black hole, while for $\beta$ positive (negative) the event horizon radius is smaller (larger) than the Schwarzschild-dS black hole, when the spacetime describes black hole solutions. 


\section{Scalar perturbations}

In order to obtain the QNMs of scalar perturbations in the background of the metric (3) we consider the Klein-Gordon equation,

$\frac{1}{\sqrt{-g}} \partial_{\mu}\left(\sqrt{-g} g^{\mu \nu} \partial_{\nu} \varphi\right)=-m^{2} \varphi$,

with suitable boundary conditions for a black hole geometry. In the above expression $m$ is the mass of the scalar field $\varphi$. Now, by means of the ansatz

$\varphi=\mathrm{e}^{-i \omega t} R(r) Y(\Omega)$,

the Klein-Gordon equation reduces to

$\frac{1}{r^{2}} \frac{\mathrm{d}}{\mathrm{d} r}\left(r^{2} B(r) \frac{\mathrm{d} R}{\mathrm{~d} r}\right)+\left(\frac{\omega^{2}}{B(r)}-\frac{\ell(\ell+1)}{r^{2}}-m^{2}\right) R(r)=0$,

where $\ell=0,1,2, \ldots$ corresponds to the eigenvalue of the Laplacian on the two-sphere. Now, defining $R(r)=\frac{F(r)}{r}$ and by using the tortoise coordinate $r^{*}$ given by $\mathrm{d} r^{*}=\frac{\mathrm{d} r}{B(r)}$, the Klein-Gordon equation can be written as

$\frac{\mathrm{d}^{2} F\left(r^{*}\right)}{\mathrm{d} r^{* 2}}-V_{\mathrm{eff}}(r) F\left(r^{*}\right)=-\omega^{2} F\left(r^{*}\right)$,

which corresponds to a one-dimensional Schrödinger-like equation with an effective potential $V_{\text {eff }}(r)$, parametrized as $V_{\text {eff }}\left(r^{*}\right)$ and given by

$V_{\mathrm{eff}}(r)=\frac{B(r)}{r^{2}}\left(\ell(\ell+1)+m^{2} r^{2}+B^{\prime}(r) r\right)$,

and substituting the metric function $B(r)$ in the effective potential in Eq. (11) we obtain

$$
\begin{aligned}
V_{\text {eff }}(r)= & \frac{1}{3}\left(3 \beta^{2}-\ell(\ell+1) \Lambda-2 \Lambda+3 m^{2}\right) \\
& +\frac{3 \beta \ell(\ell+1)+3 \beta-6 m^{2} M+2 \Lambda M}{3 r} \\
& +\frac{1}{9} r^{2}\left(2 \Lambda^{2}-3 \Lambda m^{2}\right)+r\left(\beta m^{2}-\beta \Lambda\right) \\
& -\frac{4 M^{2}}{r^{4}}+\frac{-2 \ell(\ell+1) M+2 M}{r^{3}}+\frac{\ell(\ell+1)}{r^{2}} .
\end{aligned}
$$

Now, by considering $m=m_{c}$, as the value of the mass that cancels the divergence of order $r^{2}$, i.e., $m_{c}=\sqrt{2 \Lambda / 3}$, and $\ell=0$, the effective potential is

$$
V_{\mathrm{eff}}(r)=\beta^{2}-\frac{4 M^{2}}{r^{4}}+\frac{2 M}{r^{3}}-\frac{2 \Lambda M-3 \beta}{3 r}-\frac{\beta \Lambda r}{3} .
$$

Therefore we note that for $\beta$ negative (positive) the potential diverges positive (negative) at infinity, and in principle the existence of a critical mass could not be present in this case, due to the arguments given in [68].

Now, in order to solve numerically the differential equation (9) by using the pseudospectral Chebyshev method [82], it is convenient to perform the change of variable $y=\left(r-r_{H}\right) /\left(r_{\Lambda}-r_{H}\right)$. Thus, the values of the radial coordinate are limited to the range $[0,1]$, and the radial equation (9) can be written as

$$
\begin{aligned}
& B(y) R^{\prime \prime}(y)+\left(\frac{2\left(r_{\Lambda}-r_{H}\right) B(y)}{r_{H}+\left(r_{\Lambda}-r_{H}\right) y}+B^{\prime}(y)\right) R^{\prime}(y) \\
& +\left(r_{\Lambda}-r_{H}\right)^{2}\left(\frac{\omega^{2}}{B(y)}-\frac{\ell(\ell+1)}{\left(r_{H}+\left(r_{\Lambda}-r_{H}\right) y\right)^{2}}-m^{2}\right) R(y)=0 .
\end{aligned}
$$

Also, in order to propose an ansatz for $R(y)$ we consider its behavior in the vicinity of the horizon $(\mathrm{y} \rightarrow 0)$,

$R(y)=C_{1} \mathrm{e}^{-\frac{i \omega\left(r_{\Lambda}-r_{H}\right)}{B^{\prime}(0)} \ln y}+C_{2} \mathrm{e}^{\frac{i \omega\left(r_{\Lambda}-r_{H}\right)}{B^{\prime}(0)} \ln y}$,

and at the cosmological horizon $(y=1)$,

$R(y)=D_{1} \mathrm{e}^{-\frac{i \omega\left(r_{\Lambda}-r_{H}\right)}{B^{\prime}(1)} \ln (1-y)}+D_{2} \mathrm{e}^{\frac{i \omega\left(r_{\Lambda}-r_{H}\right)}{B^{\prime}(1)} \ln (1-y)}$.

So, an ansatz that satisfies the requirements of only ingoing waves at the event horizon and at the cosmological horizon is

$R(y)=\mathrm{e}^{-\frac{i \omega\left(r_{\Lambda}-r_{H}\right)}{B^{\prime}(0)} \ln y} \mathrm{e}^{\frac{i \omega\left(r_{\Lambda}-r_{H}\right)}{B^{\prime}(1)} \ln (1-y)} F(y)$.

Then, by inserting the above ansatz for $R(y)$ in Eq. (14), it is possible to obtain an equation for the function $F(y)$. The solution for the function $F(y)$ is assumed to be a finite linear combination of the Chebyshev polynomials, then it is inserted in the differential equation for $F(y)$. Also, the interval $[0,1]$ is discretized at the Chebyshev collocation points. Then the differential equation is evaluated at each collocation point. Thus, a system of algebraic equations is obtained, and it corresponds to a generalized eigenvalue problem, which is solved numerically to obtain the QNFs. It is worth mentioning that, for $\beta=0$, the spacetime is described by the Schwarzschild-de Sitter black hole. The complex family of QNMs for this geometry was determined in [83] by using the WKB and Pöschl-Teller method. Also, it was shown that the frequencies all have a negative imaginary part, which means that the propagation of scalar field is stable in this background, and the presence of the cosmologicalconstant 

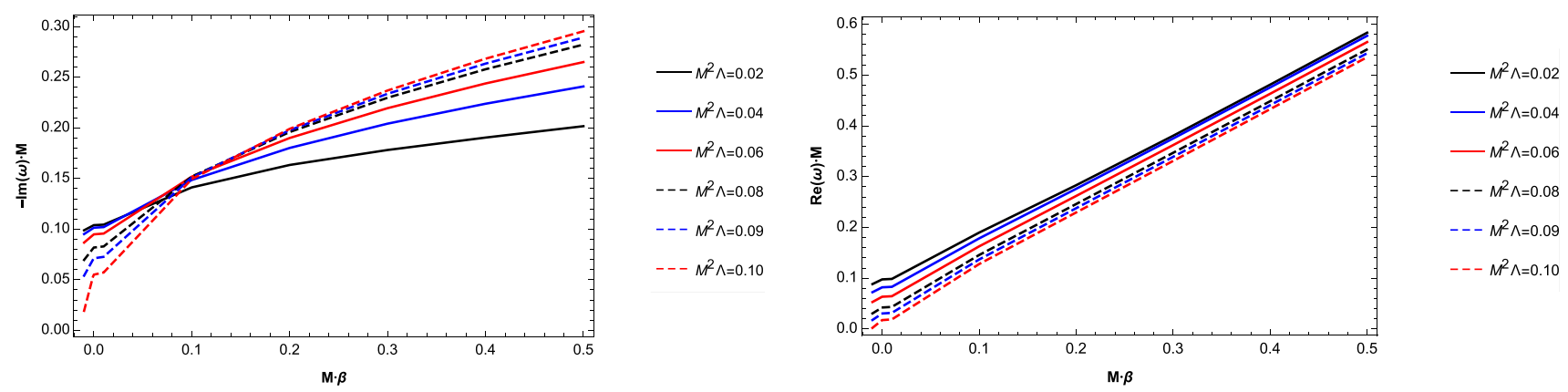

Fig. 2 The behavior of $\operatorname{Im}(\omega) M$ (left panel) and $\operatorname{Re}(\omega) M$ (right panel) for the fundamental QNF as a function of $M \beta$ for massless scalar field with $\ell=0$ and different values of the cosmological constant
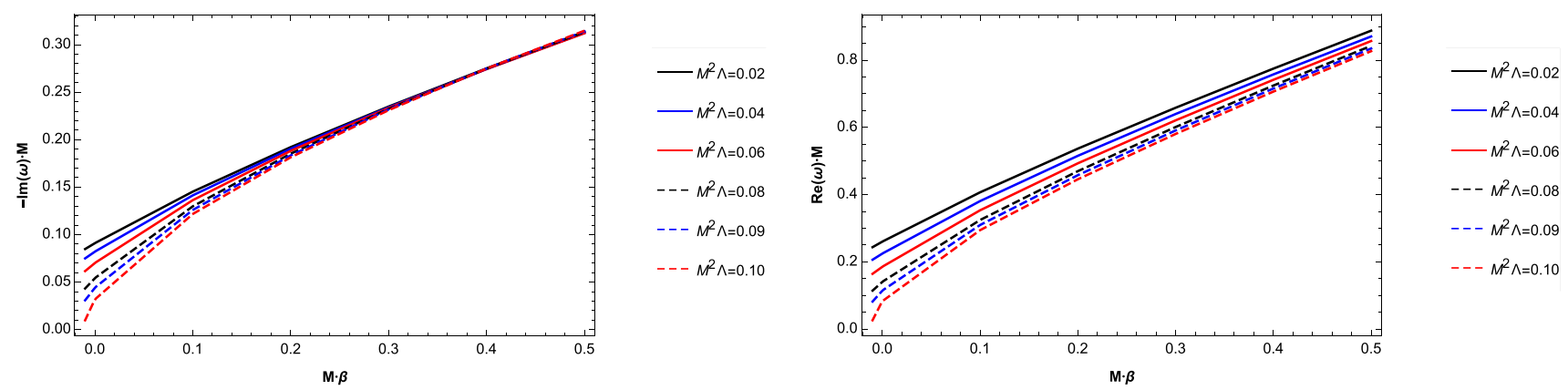

Fig. 3 The behavior of $\operatorname{Im}(\omega) M$ (left panel) and $\operatorname{Re}(\omega) M$ (right panel) for the fundamental QNF as a function of $M \beta$ for massless scalar field with $\ell=1$ and different values of the cosmological constant

leads to decrease of the real oscillation frequency and to a slower decay. High overtones were studied in [84]. Also, a family of purely imaginary modes were reported in [85] and an analysis of the photon sphere (PS) modes and de Sitter (dS) modes was recently performed in Ref. [68].

\subsection{The photon sphere family}

\subsubsection{Photon sphere modes}

The photon sphere QNMs are represented by damped oscillations whose decay rate $\left(\omega_{I}\right)$ is directly connected to the instability timescale of null geodesics at the photon sphere. The photon sphere is a spherical trapping region of space where gravity is strong enough so that photons are forced to travel in unstable circular orbits around a black hole. This region has a strong pull in the control of decay of perturbations and the QNMs with large frequencies. For instance, the decay timescale is related to the instability timescale of null geodesics near the photon sphere. For asymptotically dS black holes we find a family that can be traced back to the photon sphere and refer to them as PS modes. The dominant modes of this family are approached in the eikonal limit, where $\ell \rightarrow \infty$, for the mass of the scalar field lower than a critical value, and can be very well approximated with the WKB method. For this family of modes, higher angular momentum represents smaller decay rates, such that in the range of interest, the most representative modes are those for which $\ell \rightarrow \infty[86,87]$.

Now, in order to observe the behavior of photon sphere modes, we plot the behavior of the fundamental QNFs $n_{P S}=0^{1}$ of massless scalar fields as a function of $M \beta$ with $\ell=0$; see Fig. 2. We can observe that, for small values of $M \beta$, the decay rate decreases when the cosmological constant increases. However, for large $M \beta$, the behavior is opposite, i.e., the decay rate increases. On the other hand, the frequency of the oscillation increases when $M \beta$ increases and decreases when the cosmological constant increases. In Fig. 3 we consider $\ell=1$; note that the behavior of the QNFs is equivalent to the observed for $\ell=0$ and small $M \beta$; however, there is no inverted behavior, for the range of $M \beta$ values considered. Thus, when the cosmological constant increases the decay rate decreases, for small $M \beta$ and when $M \beta$ increases the decay rate converges to the same value and it does not depend on the value of the cosmological constant. On the other hand, the frequency of the oscillation increases when

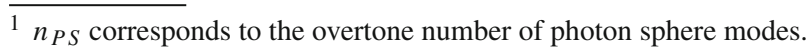



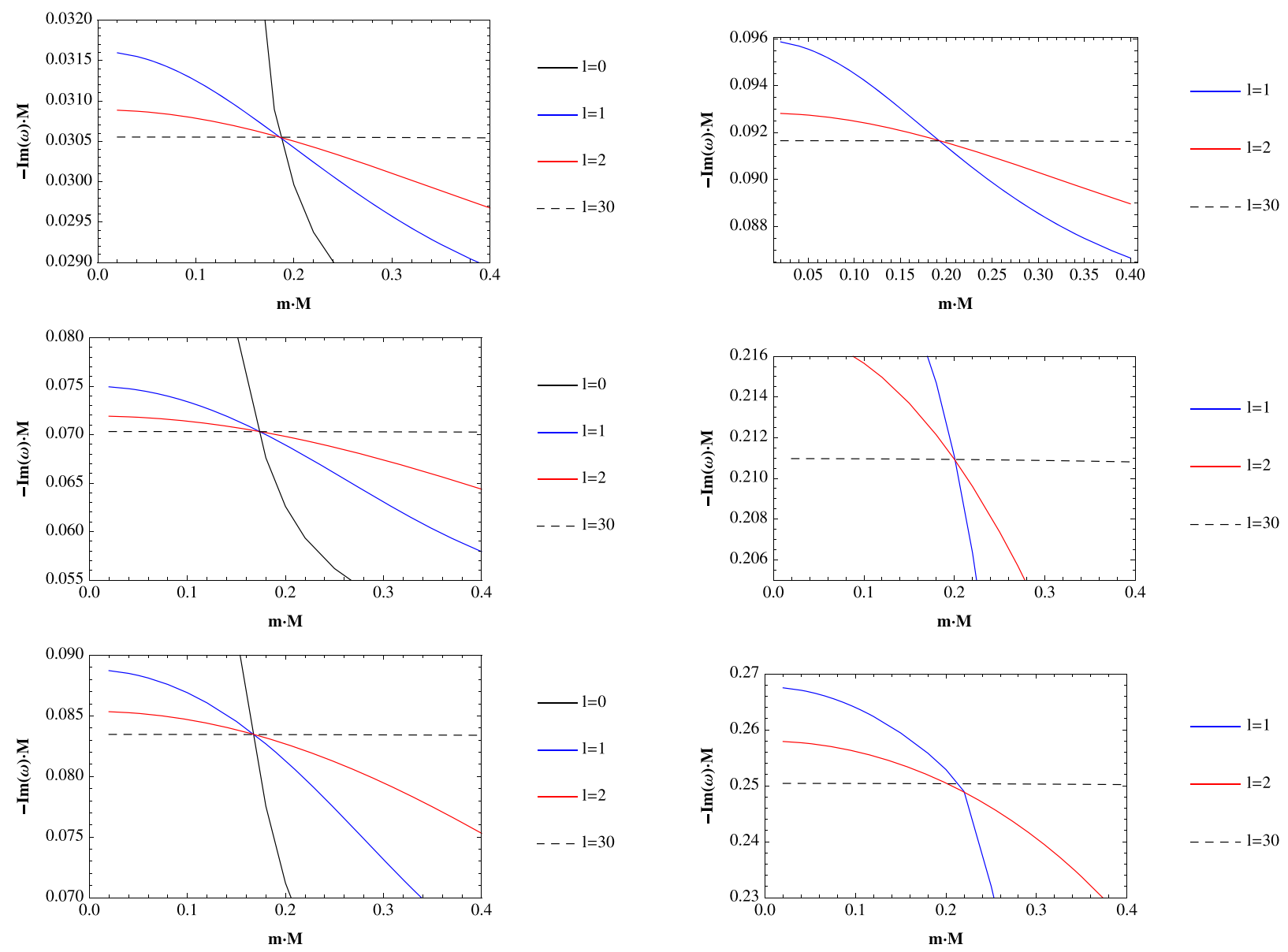

Fig. 4 The behavior of $-\operatorname{Im}(\omega) M$ as a function of the scalar field mass for different values of the parameter $\ell=0,1,2,30$ and $n_{\mathrm{PS}}=0$ (left panels), and $\ell=1,2,30$ and $n_{\mathrm{PS}}=1$ (right panels) with $M^{2} \Lambda=0.04$, and from top to bottom $M \beta=-0.06,-0.01$, and 0.01 , respectively

$M \beta$ increases and decreases when the cosmological constant increases, and the same behavior was observed for $\ell=0$.

\subsubsection{Anomalous decay rate}

Now, we will study if in $f(R)$ modified gravity, in the presence of a cosmological constant, the photon sphere modes show an anomalous behavior, i.e. the longest-lived modes are the ones with higher angular number, and also the presence of a critical mass, where beyond this value the behavior mentioned is inverted. So, we plot the behavior of $-\operatorname{Im}(\omega) M$ as a function of the scalar field mass for different values of the parameters $M \beta, \ell$ and $n_{\mathrm{PS}}=0$ (left panels), and $n_{\mathrm{PS}}=1$ with $\ell>n_{\mathrm{PS}}$ (right panels), ${ }^{2}$ for a fixed values of the black hole mass and the cosmological constant; see Fig. 4. Also, we consider larger values of the parameter $M \beta$

\footnotetext{
${ }^{2}$ We have left outside the case for $\ell=0$, because the imaginary part of the QNFs exhibits a different behavior.
}

with $n_{\mathrm{PS}}=0$; see Fig. 5 . We can observe that the anomalous behavior along with the critical mass can be present for a certain range of values of the parameter $M \beta$. See, Fig. 4, where the anomalous behavior occurs, and the critical mass appears, for $M \beta=-0.06,-0.01$, and 0.01 ; and see Fig. 5, where the anomalous behavior does occur for $M \beta=0.10$ and it does not occur and the critical mass does not appear, for $M \beta=0.15$, and 0.20 . Note that, for $M \beta=0.20$, the longest-lived modes always are the ones with smaller angular number, but for $M \beta=0.15$, we cannot say the same.

As mentioned, when $M \beta$ increases the metric function could represents naked singularity, extremal black hole, near extremal black hole and black hole solutions. So, one of the effects of $M \beta$ is tuning the different cases, which have an important effect on the anomalous behavior, that is, for the near extremal case; see Fig. 4, top panel, the value of the critical mass is the same for the overtone numbers $n_{\mathrm{PS}}=0$ and $n_{\mathrm{PS}}=1$, which does not occur for the other case where the value of the critical mass increases when the overtone 

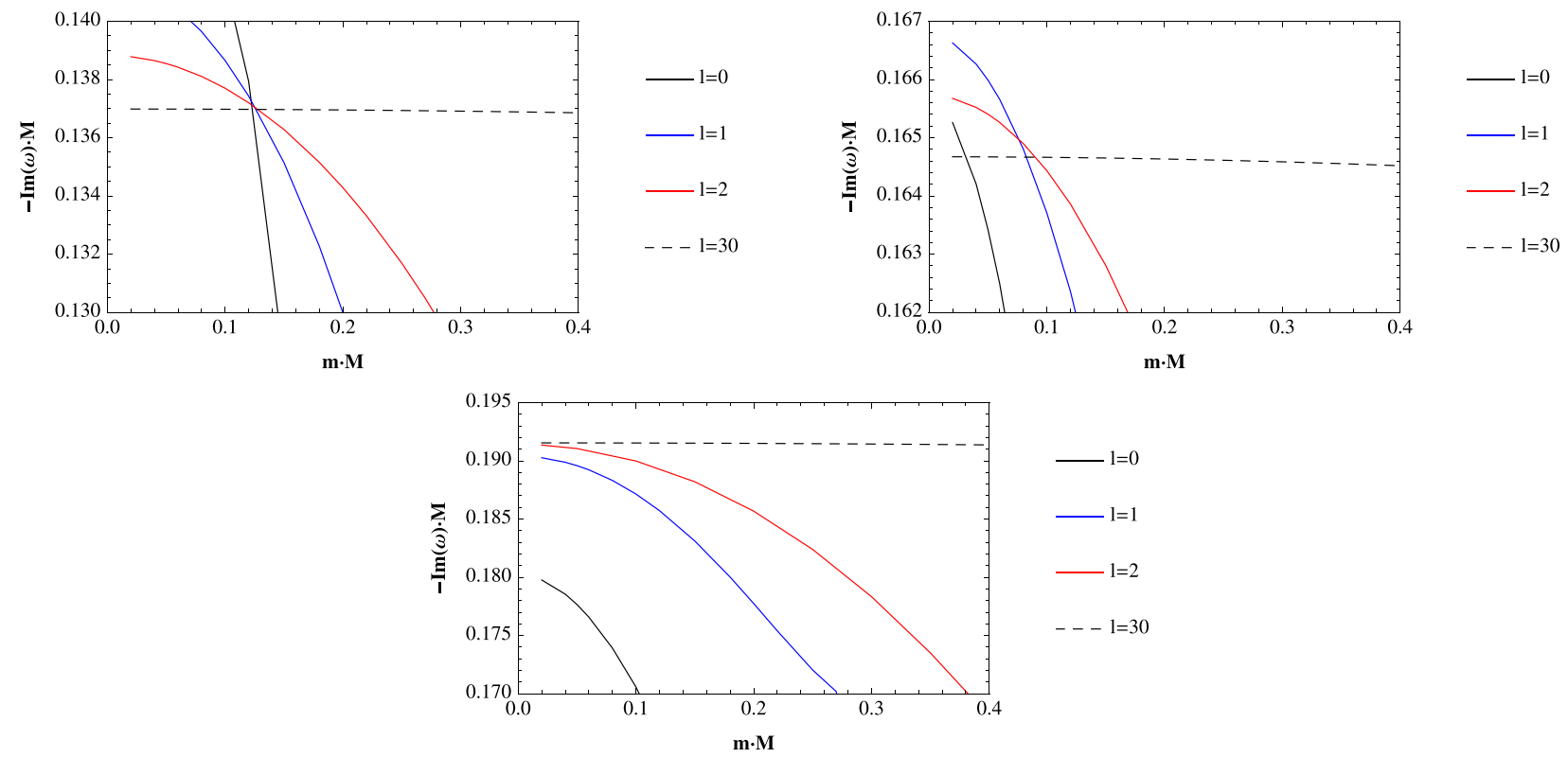

Fig. 5 The behavior of $-\operatorname{Im}(\omega) M$ as a function of the scalar field mass $m M$ for different values of the parameter $\ell=0,1,2,30$ and $n$ PS $=0$ with $M^{2} \Lambda=0.04$, and $M \beta=0.1$ (top panel), $M \beta=0.15$ (center panel) and $M \beta=0.20$ (bottom panel)

number increases. Also, we can observe that when the $M \beta$ parameter increases, the critical mass is shifted by the effect of the $M \beta$ parameter, and is decreasing. So, there is a critical value of $M \beta$ where the critical mass is zero; thus there is no anomalous behavior in the QNMs for larger values of $M \beta$.

To explain this effect, we could say that the deviation of Schwarzschild-dS black hole, where the anomalous behavior and the critical mass have been observed, is given by the parameter $M \beta$ which also appears in the metric function. So for small values of this parameter, i.e. small deviations Schwarzschild-dS black hole, the anomalous behavior occurs and the critical mass appears. However, for larger values of the parameter $M \beta$ i.e large deviations from Schwarzschild-dS black hole, the QNMs show a different behavior, where the anomalous behavior and the critical mass are not present, this cut off point could numerically occur for $M \beta \approx 0.10$; see Fig. 2, where the QNFs present an inverted behavior with respect to $M \beta$ for massless scalar field.

\subsubsection{Analysis using the WKB method}

In this section, we use the method based on WentzelKramers-Brillouin (WKB) in order to get some analytical insight of the behavior of the QNFs in the eikonal limit $\ell \rightarrow \infty$ [88-93]. It is worth mentioning that this method can be used for effective potentials which have the form of potential barriers that approach a constant value at the horizon and spatial infinity [55]. The method considers the behavior of the effective potential near its maximum value $r_{\text {max }}^{*}$. So, by using a Taylor series expansion, the potential around its maximum is given by

$V\left(r^{*}\right)=V\left(r_{\max }^{*}\right)+\sum_{i=2}^{i=\infty} \frac{V^{(i)}}{i !}\left(r^{*}-r_{\max }^{*}\right)^{i}$,

where

$V^{(i)}=\left.\frac{\mathrm{d}^{i}}{\mathrm{~d} r^{* i}} V\left(r^{*}\right)\right|_{r^{*}=r_{\text {max }}^{*}}$

corresponds to the $i$ th derivative of the potential with respect to $r^{*}$ evaluated at the maximum of the potential $r_{\max }^{*}$. Using the WKB approximation up to sixth order the QNFs are given by the following expression [94]:

$\omega^{2}=V\left(r_{\max }^{*}\right)-2 i U$,

where

$$
\begin{aligned}
U= & N \sqrt{-V^{(2)} / 2}+\frac{i}{64} \\
& \times\left(-\frac{1}{9} \frac{V^{(3) 2}}{V^{(2) 2}}\left(7+60 N^{2}\right)+\frac{V^{(4)}}{V^{(2)}}\left(1+4 N^{2}\right)\right) \\
& +\frac{N}{2^{3 / 2} 288}\left(\frac{5}{24} \frac{V^{(3) 4}}{\left(-V^{(2)}\right)^{9 / 2}}\left(77+188 N^{2}\right)\right.
\end{aligned}
$$




$$
\begin{aligned}
& +\frac{3}{4} \frac{V^{(3) 2} V^{(4)}}{\left(-V^{(2)}\right)^{7 / 2}}\left(51+100 N^{2}\right) \\
& +\frac{1}{8} \frac{V^{(4) 2}}{\left(-V^{(2)}\right)^{5 / 2}}\left(67+68 N^{2}\right) \\
& \left.+\frac{V^{(3)} V^{(5)}}{\left(-V^{(2)}\right)^{5 / 2}}\left(19+28 N^{2}\right)+\frac{V^{(6)}}{\left(-V^{(2)}\right)^{3 / 2}}\left(5+4 N^{2}\right)\right),
\end{aligned}
$$

and $N=n_{\mathrm{PS}}+1 / 2$, with $n_{P S}=0,1,2, \ldots$, is the overtone number. Now, by defining $L^{2}=\ell(\ell+1)$, we find that, for large values of $\ell$, the maximum of the potential is approximately at

$r_{\max } \approx r_{0}+\frac{1}{L^{2}} r_{1}+\mathcal{O}\left(L^{-4}\right)$,

where

$$
\begin{aligned}
V_{1} \approx & -\frac{\left(2+9 M^{2}\left(3 m^{2}-2 \Lambda\right)\right)\left(-1+9 \Lambda M^{2}\right)}{81 M^{2}} \\
& +\left(\frac{10}{27 M}+M\left(2 m^{2}-\frac{8 \Lambda}{3}\right)+3 M^{3}\left(3 m^{2}-2 \Lambda\right) \Lambda\right) \beta \\
& +\left(\frac{23}{18}+\frac{1}{4} M^{2}\left(-3 m^{2}\left(4+45 \Lambda M^{2}\right)+2 \Lambda\left(8+45 \Lambda M^{2}\right)\right)\right) \beta^{2} \\
& +\frac{1}{4} M\left(-2+3 M^{2}\left(3 m^{2}\left(4+63 \Lambda M^{2}\right)\right.\right. \\
& \left.\left.-2 \Lambda\left(8+63 \Lambda M^{2}\right)\right)\right) \beta^{3}+\mathcal{O}\left(\beta^{4}\right) .
\end{aligned}
$$

The second derivative of the potential evaluated at $r_{\text {max }}^{*}$ yields the expression

$$
V^{(2)}\left(r_{\max }^{*}\right) \approx V_{0}^{(2)} L^{2}+V_{1}^{(2)}+\mathcal{O}\left(L^{-2}\right)
$$

where

$$
\begin{aligned}
V_{0}^{(2) \approx} & -\frac{2\left(1-9 \Lambda M^{2}\right)^{2}}{729 M^{4}}-\frac{2\left(\left(-7+9 \Lambda M^{2}\right)\left(-1+9 \Lambda M^{2}\right)\right)}{243 M^{3}} \beta+\left(-\frac{32}{81 M^{2}}+\frac{13 \Lambda}{9}+\Lambda^{2} M^{2}\right) \beta^{2} \\
& -\frac{\left(8+3 \Lambda M^{2}\left(2+9 \Lambda M^{2}\right)\right)}{9 M} \beta^{3}+\mathcal{O}\left(\beta^{4}\right), \\
V_{1}^{(2) \approx} & \frac{2\left(1-9 \Lambda M^{2}\right)^{2}\left(-8+9 M^{2}\left(m^{2}\left(6-135 \Lambda M^{2}\right)+2 \Lambda\left(-1+45 \Lambda M^{2}\right)\right)\right)}{6561 M^{4}} \\
& +\frac{2\left(-1+9 \Lambda M^{2}\right)\left(64+27 M^{2}\left(4 \Lambda-54 \Lambda^{2} M^{2}\left(3+11 \Lambda M^{2}\right)+m^{2}\left(-16+9 \Lambda M^{2}\left(23+99 \Lambda M^{2}\right)\right)\right)\right)}{2187 M^{3}} \beta \\
& +\frac{\left(-116+9 M^{2}\left(-3 m^{2}\left(-25+9 \Lambda M^{2}\left(13+18 \Lambda M^{2}\left(8+63 \Lambda M^{2}\right)\right)\right)+\Lambda\left(5+27 \Lambda M^{2}\left(14+3 \Lambda M^{2}\left(41+252 \Lambda M^{2}\right)\right)\right)\right)\right)}{243 M^{2}} \beta^{2} \\
& +\left(-\frac{344}{243 M}+4 M\left(m^{2}-\Lambda\right)+5 M^{3}\left(9 m^{2}-10 \Lambda\right)+6 M^{5}\left(126 m^{2}-107 \Lambda\right) \Lambda^{2}+2106 M^{7}\left(3 m^{2}-2 \Lambda\right) \Lambda^{3}\right) \beta^{3}+\mathcal{O}\left(\beta^{4}\right),
\end{aligned}
$$

$$
\begin{aligned}
r_{0} \approx & 3 M-\frac{9 M^{2}}{2} \beta+\frac{27 M^{3}}{2} \beta^{2}-\frac{405 M^{4}}{8} \beta^{3}+\mathcal{O}\left(\beta^{4}\right), \\
r_{1} \approx & -\frac{1}{3} M\left(-1+9 M^{2}\left(3 m^{2}-2 \Lambda\right)\right)\left(-1+9 \Lambda M^{2}\right) \\
& +\frac{1}{2} M^{2}\left(-1+9 M^{2}\left(3 m^{2}-2 \Lambda\right)\right)\left(-1+63 M^{2} \Lambda\right) \beta \\
& +\frac{9}{4} M^{3}\left(2+9 M^{2} \Lambda\left(13+216 M^{2} \Lambda\right)\right. \\
& \left.-27 m^{2}\left(M^{2}+108 M^{4} \Lambda\right)\right) \beta^{2} \\
& +\frac{9}{8} M^{4}\left(-35+9 M^{2}\left(-2 \Lambda\left(98+1485 \Lambda M^{2}\right)\right.\right. \\
& \left.\left.+m^{2}\left(84+4455 \Lambda M^{2}\right)\right)\right) \beta^{3}+\mathcal{O}\left(\beta^{4}\right),
\end{aligned}
$$

and

$V\left(r_{\text {max }}^{*}\right) \approx V_{0} L^{2}+V_{1}+\mathcal{O}\left(L^{-2}\right)$

where

$V_{0} \approx\left(\frac{1}{27 M^{2}}-\frac{\Lambda}{3}\right)+\frac{\beta}{3 M}+\frac{\beta^{2}}{4}-\frac{M \beta^{3}}{4}+\mathcal{O}\left(\beta^{4}\right)$, and the higher derivatives of the potential evaluated at $r_{\max }^{*}$ yield the expressions

$$
\begin{aligned}
V^{(3)}\left(r_{\max }^{*}\right) \approx & \left(-\frac{4\left(-1+9 \Lambda M^{2}\right)^{3}}{6561 M^{5}}+\frac{4\left(1-9 \Lambda M^{2}\right)^{2}}{243 M^{4}} \beta\right. \\
& +\frac{\left(-13+9 \Lambda M^{2}\right)\left(-1+9 \Lambda M^{2}\right)}{81 M^{3}} \beta^{2} \\
& +\frac{53-9 \Lambda M^{2}\left(16+9 \Lambda M^{2}\right)}{81 M^{2}} \beta^{3} \\
& \left.+\mathcal{O}\left(\beta^{4}\right)\right) L^{2}+\mathcal{O}\left(L^{0}\right), \\
V^{(4)}\left(r_{\max }^{*}\right) \approx & \left(-\frac{16\left(-1+9 \Lambda M^{2}\right)^{3}}{19683 M^{6}}\right. \\
& -\frac{16\left(1-9 \Lambda M^{2}\right)^{2}\left(-11+18 \Lambda M^{2}\right)}{6561 M^{5}} \beta \\
& +\frac{4\left(-1+9 \Lambda M^{2}\right)\left(-7+27 \Lambda M^{2}\right)}{81 M^{4}} \beta^{2} \\
& \left.+\frac{4\left(131+45 \Lambda M^{2}\left(-20+9 \Lambda M^{2}\right)\right)}{243 M^{3}} \beta^{3}+\mathcal{O}\left(\beta^{4}\right)\right) L^{2} \\
& +\mathcal{O}\left(L^{0}\right),
\end{aligned}
$$




$$
\begin{aligned}
V^{(5)}\left(r_{\max }^{*}\right) \approx & \left(-\frac{40\left(1-9 \Lambda M^{2}\right)^{4}}{59049 M^{7}}-\frac{40\left(13-9 \Lambda M^{2}\right)\left(1-9 \Lambda M^{2}\right)^{3}}{19683 M^{6}} \beta\right. \\
& +\frac{20\left(1-9 \Lambda M^{2}\right)^{2}\left(-137+9 \Lambda M^{2}\left(28+9 \Lambda M^{2}\right)\right)}{6561 M^{5}} \beta^{2} \\
& \left.+\frac{20\left(1-9 \Lambda M^{2}\right)\left(-371+9 \Lambda M^{2}\left(149+9 \Lambda M^{2}\left(5+9 \Lambda M^{2}\right)\right)\right)}{2187 M^{4}} \beta^{3}+\mathcal{O}\left(\beta^{4}\right)\right) L^{2}+\mathcal{O}\left(L^{0}\right), \\
V^{(6)}\left(r_{\max }^{*}\right) \approx & \left(-\frac{16\left(1-9 \Lambda M^{2}\right)^{4}\left(4+15 \Lambda M^{2}\right)}{177147 M^{8}}+\frac{64\left(-1+9 \Lambda M^{2}\right)^{3}\left(5+6 \Lambda M^{2}\right)}{19683 M^{7}} \beta\right. \\
& -\frac{4\left(1-9 \Lambda M^{2}\right)^{2}\left(1517+9 \Lambda M^{2}\left(-302+81 \Lambda M^{2}\right)\right)}{19683 M^{6}} \beta^{2} \\
& \left.+\frac{4\left(-1+9 \Lambda M^{2}\right)\left(15655+9 \Lambda M^{2}\left(-9161+9 \Lambda M^{2}\left(841+81 \Lambda M^{2}\right)\right)\right.}{19683 M^{5}} \beta^{3}+\mathcal{O}\left(\beta^{4}\right)\right) L^{2}+\mathcal{O}\left(L^{0}\right) .
\end{aligned}
$$

Then, using the expressions above with Eq. (20), we obtain the following analytical expression for the QNFs for large values of $\ell$ and small values of $M \beta$ :

$\omega \approx \omega_{-1} L+\omega_{0}+\omega_{1} L^{-1}+\omega_{2} L^{-2}+\mathcal{O}\left(L^{-3}\right)$,

where

$$
\begin{aligned}
& \omega_{-1} \approx \frac{\sqrt{1-9 \Lambda M^{2}}}{3 \sqrt{3} M}+\frac{\sqrt{3}}{2 \sqrt{1-9 \Lambda M^{2}}} \beta-\frac{3 \sqrt{3} M\left(2+9 \Lambda M^{2}\right)}{8\left(1-9 \Lambda M^{2}\right)^{3 / 2}} \beta^{2}+\frac{3 \sqrt{3} M^{2} \sqrt{1-9 \Lambda M^{2}}\left(-16+9 \Lambda M^{2}\left(-13+18 \Lambda M^{2}\right)\right)}{16\left(-1+9 \Lambda M^{2}\right)^{3}} \beta^{3} \\
& +\mathcal{O}\left(\beta^{4}\right) \text {, } \\
& \omega_{0} \approx-i \frac{\sqrt{1-9 \Lambda M^{2}}}{6 \sqrt{3} M}-i \frac{4-9 \Lambda M^{2}}{4 \sqrt{3} \sqrt{1-9 \Lambda M^{2}}} \beta+i \frac{3 \sqrt{3} M\left(1+9 \Lambda M^{2}\left(1+9 \Lambda M^{2}\right)\right)}{16\left(1-9 \Lambda M^{2}\right)^{3 / 2}} \beta^{2} \\
& +i \frac{3 \sqrt{3} M^{2}\left(-8+9 \Lambda M^{2}\left(-13+9 \Lambda M^{2}\left(-13+63 \Lambda M^{2}\right)\right)\right)}{32\left(1-9 \Lambda M^{2}\right)^{5 / 2}} \beta^{3} \\
& +\mathcal{O}\left(\beta^{4}\right), \\
& \omega_{1} \approx-\frac{\sqrt{1-9 \Lambda M^{2}}\left(-34+9 M^{2}\left(-108 m^{2}+61 \Lambda\right)\right)}{648 M \sqrt{3}} \\
& +\frac{314-45 \Lambda M^{2}\left(59+180 \Lambda M^{2}\right)+972 m^{2}\left(M^{2}+18 \Lambda M^{4}\right)}{432 \sqrt{3} \sqrt{1-9 \Lambda M^{2}}} \beta \\
& -\frac{-710 M-5832 M^{7}\left(135 m^{2}-46 \Lambda\right) \Lambda^{2}+108 M^{3}\left(72 m^{2}+5 \Lambda\right)+243 \Lambda M^{5}\left(396 m^{2}+13 \Lambda\right)}{576 \sqrt{3}\left(1-9 \Lambda M^{2}\right)^{3 / 2}} \beta^{2} \\
& +M^{2}\left(-1262+9 M^{2}\left(\Lambda\left(-466+27 \Lambda M^{2}\left(335+6 \Lambda M^{2}\left(301-2736 \Lambda M^{2}\right)\right)\right)\right.\right. \\
& \left.\left.+324 m^{2}\left(10+3 \Lambda M^{2}\left(31+18 \Lambda M^{2}\left(-31+126 \Lambda M^{2}\right)\right)\right)\right)\right) \beta^{3} /\left(384 \sqrt{3}\left(1-9 \Lambda M^{2}\right)^{5 / 2}\right)+\mathcal{O}\left(\beta^{4}\right), \\
& \omega_{2} \approx-i \frac{\left(137+45 M^{2}\left(-648 m^{2}+401 \Lambda\right)\right)\left(1-9 \Lambda M^{2}\right)^{3 / 2}}{23328 M \sqrt{3}} \\
& +i \frac{\sqrt{1-9 \Lambda M^{2}}\left(590+17496 m^{2} M^{2}\left(2+27 \Lambda M^{2}\right)-9 \Lambda M^{2}\left(3593+30807 \Lambda M^{2}\right)\right)}{5184 \sqrt{3}} \beta \\
& +i \frac{M\left(7949+9 M^{2}\left(648 m^{2}\left(-23+9 \Lambda M^{2}\left(-11+711 \Lambda M^{2}\right)\right)+\Lambda\left(8714-9 \Lambda M^{2}\left(7858+252747 \Lambda M^{2}\right)\right)\right)\right)}{6912 \sqrt{3} \sqrt{1-9 \Lambda M^{2}}} \beta^{2} \\
& +i M^{2}\left(-23674+81 M^{2}\left(\Lambda\left(-2219+3 \Lambda M^{2}\left(70514+627708 \Lambda M^{2}-8621073 \Lambda^{2} M^{4}\right)\right)\right.\right. \\
& \left.\left.+72 m^{2}\left(154+27 \Lambda M^{2}\left(-65+27 \Lambda M^{2}\left(-119+977 \Lambda M^{2}\right)\right)\right)\right)\right) \beta^{3} /\left(13824 \sqrt{3}\left(1-9 \Lambda M^{2}\right)^{3 / 2}\right)+\mathcal{O}\left(\beta^{4}\right) .
\end{aligned}
$$




$$
\begin{aligned}
m_{c} M \approx & \sqrt{\frac{137+18045 M^{2} \Lambda}{29160}-\frac{\left(1886-4185 M^{2} \Lambda\right) M \beta}{60 \sqrt{10} \sqrt{137+18045 M^{2} \Lambda}}} \\
& -\frac{3\left(4634227+134256255 M^{2} \Lambda+1091552625 M^{4} \Lambda^{2}\right) M^{2} \beta^{2}}{400 \sqrt{10}\left(137+18045 M^{2} \Lambda\right)^{3 / 2}} \\
& -\frac{9\left(5019824246+76442598769 M^{2} \Lambda-4248331450335 M^{4} \Lambda^{2}-85433253134175 M^{6} \Lambda^{3}\right) M^{3} \beta^{3}}{800 \sqrt{10}\left(137+18045 M^{2} \Lambda\right)^{5 / 2}}+\cdots,
\end{aligned}
$$

and it is valid for small values of $\beta$ and $n_{\mathrm{PS}}=0$. In all the above expressions we have performed a Taylor expansion around $\beta=0$ in order to obtain expressions that will be easy to handle analytically. For $\beta=0$ we recover the result of critical mass for Schwarzschild-dS black holes [68], and for $\Lambda M^{2}=0.04$ and $n_{\mathrm{PS}}=0$ we obtain from (24) the values $m_{c} M=0.186,0.175,0.168$, and 0.130 for $\beta=-0.06,-0.01,0.01$, and 0.10 , respectively, which agrees with the numerical results shown in Fig. 4. Thereby the value of the critical mass decreases when the beta parameter increases and it increases when $\Lambda M^{2}$ increases; see Fig. 6. Also, note that for small values of $\Lambda M^{2}$ there is a range of values for $M \beta$ where $m_{c} M$ becomes negative. Also, the WKB method proposes a critical scalar field mass for $M \beta=0.20$, contrary to the observed via the pseudospectral Chebyshev method. However, the analysis performed with the WKB method is valid only for small values of $\beta$, because we have performed a Taylor expansion around $\beta=0$ to obtain the formula (24).

Now, in order to check the correctness and accuracy of the pseudospectral Chebyshev method with respect to the analytical expression for the QNFs given by Eq. (23), we show in Table 1, the values obtained with the two methods. Also, we show the relative error, which is defined by

$\begin{aligned} \epsilon_{R e(\omega)} & =\frac{\left|\operatorname{Re}\left(\omega_{1}\right)\right|-\left|\operatorname{Re}\left(\omega_{0}\right)\right|}{\left|\operatorname{Re}\left(\omega_{0}\right)\right|} \cdot 100 \%, \\ \epsilon_{\operatorname{Im}(\omega)} & =\frac{\left|\operatorname{Im}\left(\omega_{1}\right)\right|-\left|\operatorname{Im}\left(\omega_{0}\right)\right|}{\left|\operatorname{Im}\left(\omega_{0}\right)\right|} \cdot 100 \%,\end{aligned}$

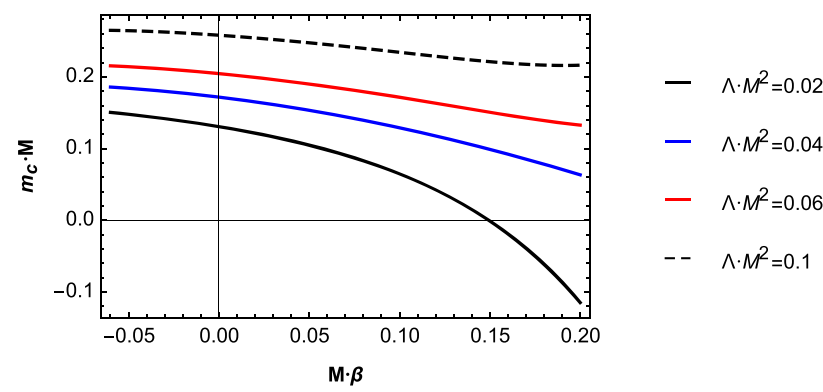

Fig. 6 The behavior of the $m_{c} M$ as a function of $M \beta$ for different values of $\Lambda M^{2}$ with $n_{\mathrm{PS}}=0$ where $\omega_{1}$ corresponds to the result from Eq. (23) and $\omega_{0}$ denotes the result with the pseudospectral Chebyshev method. We can observe that the error does not exceed 0.0008 $\%$ in the imaginary part, and $0.0010 \%$ in the real part. Also, as it was observed, the frequencies all have a negative imaginary part, which means that the propagation of massive scalar fields is stable in this background.

As we mentioned, the Taylor series have been performed around $\beta=0$, so the WKB analysis is true for small values of $\beta$. In Table 2 , we show the error in the quasinormal frequency between the WKB and pseudospectral method, for different values of $m M$, and $M \beta$. Note that for $M \beta=0.20$ the error is $18 \%$ in the imaginary part, and $31 \%$ in the real part, approximately, that mean a loss of robustness and diminished validity in the results. Also, we can observe that the error increases slightly, when $m M$ increases.

\subsection{The de Sitter family}

The de Sitter family of modes are related to the accelerated expansion of the universe, which is related to the surface gravity of the cosmological horizon of pure dS space. They correspond to purely imaginary modes which can be very well approximated by the pure dS QNMs. The pure dS modes are determined by two branches $[95,96]$, and the most important for our purposes corresponds to the lowest lying solution, given by

$\omega_{\text {pure dS }}=-i \sqrt{\frac{\Lambda}{3}}\left(2 n+\ell+\frac{3}{2} \pm \sqrt{\frac{9}{4}-3 \frac{m^{2}}{\Lambda}}\right)$

where $n$ is the overtone number.

In order to visualize the behavior of the QNFs for the $\mathrm{dS}$ modes, in Fig. 7 we plot $-\operatorname{Im}(\omega) M$ for $\ell=1$ and different values of the cosmological constant, note that there is a low decay rate when $M \beta$ increases, and when the cosmological constant decreases. Also, as before, we will study the behavior of the dS QNFs as a function of the scalar field mass. So, we obtain some QNFs for different values of the parameters $\ell$ and $n_{\mathrm{dS}}=0,{ }^{3}$ for a fixed values of the black hole mass and

\footnotetext{
${ }^{3} n_{\mathrm{dS}}$ corresponds to the overtone number of de Sitter modes
} 
Table 1 Fundamental quasinormal frequencies $\left(n_{P S}=0\right)$ for massive scalar fields with $\ell=10,20$, and 30 in the background of black hole solution of $f(R)$ gravity with $M^{2} \Lambda=0.04$, and $M \beta=0.01$

\begin{tabular}{rlrr}
\hline$\ell$ & Pseudospectral method & WKB & $\epsilon_{\operatorname{Re}(\omega)}$ \\
\hline$m M=0.02$ & & & $\epsilon_{\operatorname{Im}(\omega)}$ \\
10 & $1.72651001-0.08356503 i$ & $1.72652389-0.08356574 i$ & 0.0008 \\
20 & $3.37194090-0.08348702 i$ & $3.37197238-0.08348760 i$ & 0.0009 \\
30 & $5.01712051-0.08347182 i$ & $5.01716774-0.08347239 i$ & 0.0009 \\
$m M=0.15$ & & & 0.0008 \\
10 & $1.72802056-0.08348145 i$ & $1.72803867-0.08348207 i$ & 0.0010 \\
20 & $3.37271554-0.08346511 i$ & $3.37274759-0.08346568 i$ & 0.0010 \\
30 & $5.01764129-0.08346193 i$ & $5.01768870-0.08346249 i$ & 0.0007 \\
$m M=0.30$ & & & 0.00007 \\
10 & $1.73263370-0.08322691 i$ & $1.73266525-0.08322649 i$ & 0.0007 \\
20 & $3.37508142-0.08339825 i$ & $3.37511532-0.08339874 i$ & 0.0018 \\
30 & $5.01923188-0.08343171 i$ & $5.01927986-0.083432269$ & 0.0010 \\
\hline
\end{tabular}

Table 2 Fundamental quasinormal frequencies $\left(n_{\mathrm{PS}}=0\right)$ for massive scalar fields with $\ell=30$ in the background of black hole solution of $f(R)$ gravity with $M^{2} \Lambda=0.04$, and different values of $M \beta$

\begin{tabular}{|c|c|c|c|c|}
\hline$M \beta$ & Pseudospectral method & WKB & $\epsilon_{R e(\omega)}$ & $\epsilon_{\operatorname{Im}(\omega)}$ \\
\hline \multicolumn{5}{|c|}{$m M=0.02$} \\
\hline-0.06 & $2.08314131-0.03055262 i$ & $2.25222758-0.03267338 i$ & 8.11689 & 6.94134 \\
\hline-0.01 & $4.35540688-0.07032092 i$ & $4.35546389-0.07032160 i$ & 0.00130895 & 0.000966995 \\
\hline 0.01 & $5.01712051-0.08347182 i$ & $5.01716774-0.08347239 i$ & 0.000941377 & 0.000682865 \\
\hline 0.10 & $7.42982610-0.13698770 i$ & $7.70221565-0.14017416 i$ & 3.66616 & 2.32609 \\
\hline 0.15 & $8.55684679-0.16467397 i$ & $9.67805702-0.17771512 i$ & 13.1031 & 7.91938 \\
\hline 0.20 & $9.59615293-0.19154767 i$ & $12.58587755-0.22617900 i$ & 31.1554 & 18.0797 \\
\hline \multicolumn{5}{|c|}{$m M=0.15$} \\
\hline-0.06 & $2.08341635-0.03055125 i$ & $2.25253125-0.03267230 i$ & 8.11719 & 6.9426 \\
\hline-0.01 & $4.35588702-0.07031330 i$ & $4.35594414-0.07031399 i$ & 0.00131133 & 0.000981322 \\
\hline 0.01 & $5.01764129-0.08346193 i$ & $5.01768870-0.08346249 i$ & 0.000944866 & 0.000670965 \\
\hline 0.10 & $7.43044497-0.13696970 i$ & $7.70288435-0.14015649 i$ & 3.66653 & 2.32664 \\
\hline 0.15 & $8.55749294-0.16465262 i$ & $9.67890740-0.17769503 i$ & 13.1045 & 7.92117 \\
\hline 0.20 & $9.59681747-0.19152351 i$ & $12.58708647-0.22615793 i$ & 31.159 & 18.0836 \\
\hline \multicolumn{5}{|c|}{$m M=0.30$} \\
\hline-0.06 & $2.08425622-0.03054706 i$ & $2.25345872-0.03266898 i$ & 8.11812 & 6.9464 \\
\hline-0.01 & $4.35735346-0.07029005 i$ & $4.35741099-0.07029073 i$ & 0.0013203 & 0.00096742 \\
\hline 0.01 & $5.01923188-0.08343171 i$ & $5.01927986-0.08343226 i$ & 0.000955923 & 0.000659222 \\
\hline 0.10 & $7.43233530-0.13691473 i$ & $7.70492677-0.14010254 i$ & 3.66764 & 2.32832 \\
\hline 0.15 & $8.55946661-0.16458739 i$ & $9.68150472-0.17763367 i$ & 13.1087 & 7.92666 \\
\hline 0.20 & $9.59884736-0.19144971 i$ & $12.59077885-0.22609358 i$ & 31.1697 & 18.0955 \\
\hline
\end{tabular}

the cosmological constant; see Table 3 for $M \beta=0.01$. As we mentioned, this family is dominant for small masses and it can render a real part becoming complex, which depends on the scalar field mass and the angular number. Also, it is possible to observe in Table 3 that the decay rate always increases when the angular number increases, thereby the longest-lived modes are the ones with lower angular number and the anomalous behavior is not present in this family.

3.3 The dominance family

In order to observe the dominance between the photon and de Sitter family we plot in Fig. 8 the imaginary part of the QNFs 


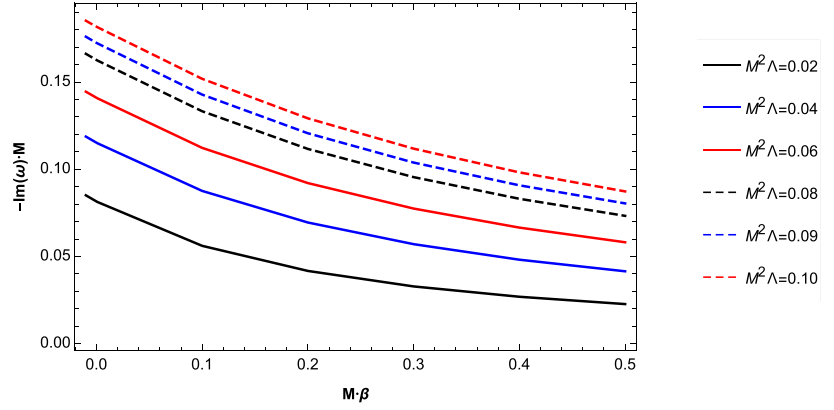

Fig. 7 The behavior of the fundamental dS mode $\left(n_{d S}=0\right)$ $-\operatorname{Im}(\omega) M$ as a function of $M \beta$ obtained by using the pseudospectral Chebyshev method for massless scalar field with $\ell=1$ and different values of the cosmological constant

as a function of the scalar field mass, for different overtone numbers and $\ell=0$, where the black points correspond to the purely imaginary QNFs, and the gray color points correspond to the complex QNFs for small values of the parameter $M \beta=$ 0.01. The behavior is similar to that observed in Ref. [68]. We can recognize the two families for zero mass of the scalar field, a family of complex QNFs, and a purely imaginary family. As we have seen, the purely imaginary modes belong to the family of de Sitter modes, while the complex ones correspond to the photon sphere modes.

In order to interpret this figure, first observe the black points, and $n_{\mathrm{dS}}=0$. This family is dominant for small masses and near $m M=0.17$ it can render a real part becoming complex due to the continuity between the black and gray points, and also could be connected with the $\mathrm{dS}$ modes with $-\operatorname{Im}(\omega) M \approx 1$. Also, for low values of the mass, it is possible to observe that, for $n_{\mathrm{dS}}=1$ and $n_{\mathrm{dS}}=2$, one can have a combination rendering a real part complex for $m M \approx 0.05$; the same occurs for the overtone numbers
$n_{\mathrm{dS}}=3$ and $n_{\mathrm{dS}}=4$, and for $m M \approx 0.025$ becoming complex. Besides, for higher overtone numbers, there are dS modes purely imaginary for the whole range of mass considered. On the other hand, for the photon sphere modes, the gray points, we can see that for $n_{\mathrm{PS}}=0$ and small mass this branch is subdominant; however, for $m M \approx 0.155$ it begins to dominate.

Now, we consider a bigger value of the parameter, $M \beta=$ 0.20; see Fig. 9. As before, the black points correspond to the purely imaginary QNFs, and the gray points correspond to the complex QNFs. Note that for low values of the scalar field mass dS modes are dominant; however for larger mass the photon sphere modes are dominant. Also, it is possible to observe that dS modes with $n_{\mathrm{dS}}=0$ could to connect with $\mathrm{dS}$ modes with $n_{\mathrm{dS}}=1(-\operatorname{Im}(\omega) M \approx 1.10)$. Besides, for higher overtones numbers, there is a purely imaginary family of dS modes for the whole range of mass considered. Also, for the photon sphere modes, the gray points, we can see that for $n_{\mathrm{PS}}=0$ and small mass this family is subdominant; however, for $m M \approx 0.13$ it begins to dominate.

\section{Conclusions}

In this work, we considered an asymptotically de Sitter black hole solution with a specific $f(R)$ modified gravity as background. In order to see if strong curvature effects, which arise from non-linear terms in $R$ in the action, can modify the behavior of the QNMs, we studied the propagation of a probe scalar field and we analyzed the presence of anomalous behavior in the quasinormal modes spectrum. First, we showed that the QNM spectra consist of two families: the photon sphere modes and the de Sitter modes, and their

Table 3 Fundamental dS modes $\left(n_{\mathrm{dS}}=0\right)$ for massive scalar fields with $\ell=0,1,2$ in the background of black hole solution of $f(R)$ gravity with $M^{2} \Lambda=0.04$, and $M \beta=0.01$

\begin{tabular}{llll}
\hline$\ell$ & $m M=0.02$ & $m M=0.05$ & $m M=0.10$ \\
\hline 0 & $-0.00096728 i$ & $-0.00623134 i$ & $-0.02841651 i$ \\
1 & $-0.11303750 i$ & $-0.11914334 i$ & $-0.14367078 i$ \\
2 & $-0.22668753 i$ & $-0.23290179 i$ & $0.39967626-0.25614428 i$ \\
\hline$\ell$ & $m M=0.15$ & $m M=0.20$ & $m M=0.25$ \\
\hline 0 & $-0.09297689 i$ & $0.09799882-0.17514088 i$ & $0.14338038-0.17214279 i$ \\
1 & $-0.20354608 i$ & $0.23373131-0.25290461 i$ & $0.22754315-0.23202223 i$ \\
2 & $0.40221808-0.25380624 i$ & $0.40581565-0.25048547 i$ & $0.41051976-0.24611626 i$ \\
\hline$\ell$ & $m M=0.30$ & $m M=0.35$ & $m M=0.40$ \\
\hline 0 & $0.17714386-0.16988129 i$ & $0.20756224-0.16809914 i$ & $0.23661390-0.16676332 i$ \\
1 & $0.25091348-0.20524307 i$ & $0.27737304-0.19237303 i$ & $0.30313974-0.18446717 i$ \\
2 & $0.41643654-0.24058189 i$ & $0.42382467-0.23371594 i$ & $0.43324930-0.22546868 i$
\end{tabular}




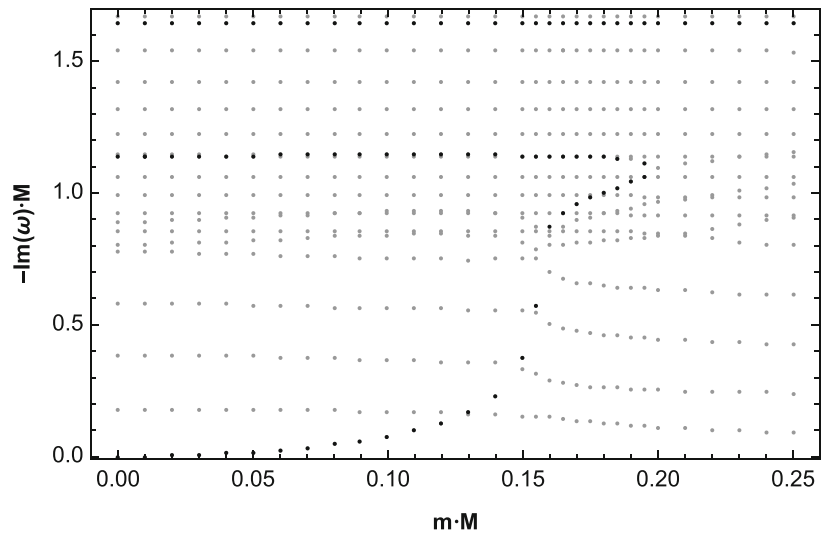

Fig. 8 The behavior of the imaginary part of the quasinormal frequencies $-\operatorname{Im}(\omega) M$ as a function of the scalar field mass $m M$ for different overtone numbers with $\ell=0, M \beta=0.01$, and $M^{2} \Lambda=0.04$. Black points are for purely imaginary QNFs and gray points for complex QNFs. Here, different colors do not describe the families, except for $m M=0$, where the black points correspond to the dS family, while the gray points correspond to the photon sphere family. Then, in order to distinguish the family for $m M>0$ it is necessary to connect it with $m M=0$, where the different families have been defined

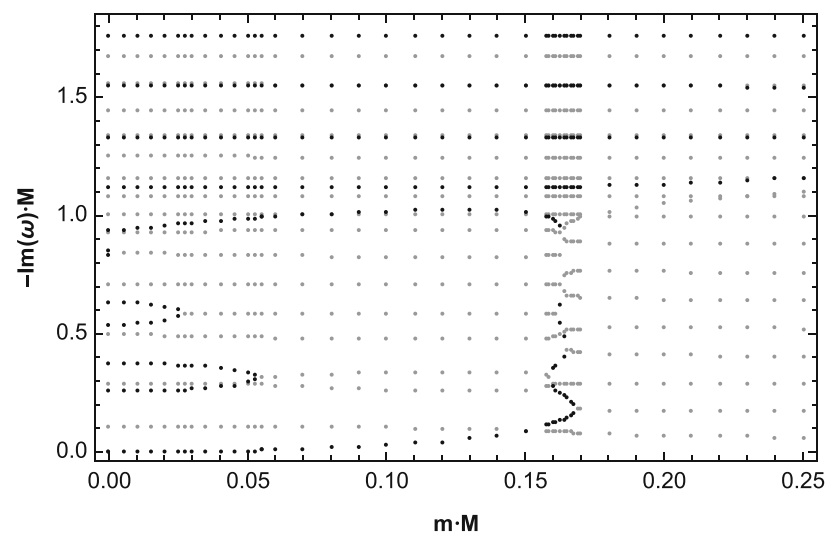

Fig. 9 The behavior of the imaginary part of the quasinormal frequencies $-\operatorname{Im}(\omega) M$ as a function of the scalar field mass $m M$ for different overtone numbers with $\ell=0, M \beta=0.20$, and $M^{2} \Lambda=0.04$. Black points for purely imaginary QNFs and gray points for complex QNFs. Here, different colors do not describe the families, except for $m M=0$, where the black points correspond to the dS family, while the gray points correspond to the photon sphere family. Then, in order to distinguish the family for $m M>0$ it is necessary to connect it with $m M=0$, where the different families have been defined

behavior depends on the parameter $\beta$, which characterizes the considered black hole solution of the $f(R)$ gravity.

For the photon sphere modes we found that, for massless scalar field and small values of the parameter $M \beta$, the decay rate decreases when the cosmological constant increases; this behavior is similar to the observed for Schwarzschild- $\mathrm{dS}$ black holes [68]. On the other hand, for large values of the parameter $M \beta$ the behavior is inverted, i.e. the decay rate increases when the cosmological constant increases.
Also, we found that the photon sphere modes show an anomalous behavior that depends on the value of the parameter $\beta$ and the mass of the scalar field. As in the case of the Schwarzschild-dS black holes, which corresponds to $\beta=0$, there is a critical value of the mass of the scalar field, which depends on the cosmological constant, beyond which the QNMs show an anomalous behavior. However, in $f(R)$ gravity this critical value depends not only on cosmological constant but also on the new parameter $\beta$, which expresses the presence of non-linear terms in the curvature. We found that the critical value of the scalar mass decreases when the parameter $M \beta$ increases, and there is a critical value of $M \beta$ where the critical mass is zero indicating that there is no anomalous behavior of the QNMs. Also, we saw that the critical value of the mass of the scalar field increases when the overtone number increases; however, when the parameter $M \beta$ approaches a near extremal black hole, i.e. when the cosmological horizon approximates to the event horizon, the critical value of the mass of the scalar field does not depend of the overtone number.

On the other hand, for the dS modes we observed that for a massless scalar field they are purely imaginary, and for massive scalar field they can render an oscillatory part becoming complex for some value of the mass and higher. Also, we did not find an anomalous behavior for this family of modes.

Also, we found that the imaginary part of the quasinormal frequencies is always negative, leading to a stable propagation of the scalar fields in this background for both families of modes. The PS family or the dS family may dominate the evolution of the perturbation depending on the values of the parameters. We showed that for small values of $M^{2} \Lambda$ the dS modes dominate for small values of the scalar field mass, while the photon sphere modes dominate for larger values of the scalar field mass. Also, the decay rate of the PS modes increases with $M \beta$, while the decay rate of the dS modes decreases, so it can occur that for low values of $M \beta$ the PS modes dominate and for high values of $M \beta$ the dS modes dominate, depending on the value of $M^{2} \Lambda$.

An interesting extension of this work is to calculate the QNMs and QNFs of the $f(R)$ gravity theory if we allow the matter fields to backreact with the metric. Then we can study the properties of the resulting hairy black holes [78, 79] and possibly see the interplay effects of the geometric scalar present in the $f(R)$ theory and the matter scalar field minimally coupled to the modified gravity theory.

Acknowledgements We thank the referee for his/her careful review of the manuscript and his/her valuable comments and suggestions. P. A. G. acknowledges the hospitality of the Universidad de La Serena where part of this work was undertaken. Y.V. acknowledge support by the Dirección de Investigación y Desarrollo de la Universidad de La Serena, Grant No. PR18142. 
Data Availability Statement This manuscript has no associated data or the data will not be deposited. [Authors' comment: This is a theoretical paper without associated data.]

Open Access This article is licensed under a Creative Commons Attribution 4.0 International License, which permits use, sharing, adaptation, distribution and reproduction in any medium or format, as long as you give appropriate credit to the original author(s) and the source, provide a link to the Creative Commons licence, and indicate if changes were made. The images or other third party material in this article are included in the article's Creative Commons licence, unless indicated otherwise in a credit line to the material. If material is not included in the article's Creative Commons licence and your intended use is not permitted by statutory regulation or exceeds the permitted use, you will need to obtain permission directly from the copyright holder. To view a copy of this licence, visit http://creativecomm ons.org/licenses/by/4.0/.

Funded by $\mathrm{SCOAP}^{3}$.

\section{References}

1. S. Nojiri, S.D. Odintsov, Introduction to modified gravity and gravitational alternative for dark energy. Conf. C 0602061, 06 (2006). arXiv:hep-th/0601213

2. S. Nojiri, S.D. Odintsov, Introduction to modified gravity and gravitational alternative for dark energy. Int. J. Geom. Methods Mod. Phys. 4, 115 (2007)

3. E.J. Copeland, M. Sami, S. Tsujikawa, Dynamics of dark energy. Int. J. Mod. Phys. D 15, 1753 (2006). arXiv:hep-th/0603057

4. S. Nojiri, S.D. Odintsov, Unified cosmic history in modified gravity: from $\mathrm{F}(\mathrm{R})$ theory to Lorentz non-invariant models. Phys. Rep. 505, 59 (2011). arXiv:1011.0544 [gr-qc]

5. T. Clifton, P.G. Ferreira, A. Padilla, C. Skordis, Modified gravity and cosmology. Phys. Rep. 513, 1 (2012). arXiv: 1106.2476 [astroph.CO]

6. S. Capozziello, M. Francaviglia, Extended theories of gravity and their cosmological and astrophysical applications. Gen. Relativ. Gravit. 40, 357-420 (2008). arXiv:0706.1146 [astro-ph]

7. A. De Felice, S. Tsujikawa, $\mathrm{f}(\mathrm{R})$ theories. Living Rev. Relativ. 13, 3 (2010). arXiv: 1002.4928 [gr-qc]

8. G. Cognola, E. Elizalde, S. Nojiri, S.D. Odintsov, L. Sebastiani, S. Zerbini, A class of viable modified $\mathrm{f}(\mathrm{R})$ gravities describing inflation and the onset of accelerated expansion. Phys. Rev. D 77, 046009 (2008). arXiv:0712.4017 [hep-th]

9. L. Pogosian, A. Silvestri, The pattern of growth in viable $f(R)$ cosmologies. Phys. Rev. D 77, 023503 (2008). Erratum: [Phys. Rev. D 81, 049901 (2010)]. arXiv:0709.0296 [astro-ph]

10. P. Zhang, Testing $f(R)$ gravity against the large scale structure of the universe. Phys. Rev. D 73, 123504 (2006). arXiv:astro-ph/0511218

11. B. Li, J.D. Barrow, The cosmology of $f(R)$ gravity in metric variational approach. Phys. Rev. D 75, 084010 (2007). arXiv:gr-qc/0701111

12. Y.S. Song, H. Peiris, W. Hu, Cosmological constraints on f(R) acceleration models. Phys. Rev. D 76, 063517 (2007). arXiv:0706.2399 [astro-ph]

13. S. Nojiri, S.D. Odintsov, Modified $\mathrm{f}(\mathrm{R})$ gravity unifying $\mathrm{R}^{* *} \mathrm{~m}$ inflation with Lambda CDM epoch. Phys. Rev. D 77, 026007 (2008). arXiv:0710.1738 [hep-th]

14. S. Nojiri, S.D. Odintsov, Unifying inflation with LambdaCDM epoch in modified $\mathrm{f}(\mathrm{R})$ gravity consistent with Solar System tests. Phys. Lett. B 657, 238 (2007). arXiv:0707.1941 [hep-th]
15. S. Capozziello, C.A. Mantica, L.G. Molinari, Cosmological perfect-fluids in $\mathrm{f}(\mathrm{R})$ gravity. Int. J. Geom. Methods Mod. Phys. 16(01), 1950008 (2018). arXiv:1810.03204 [gr-qc]

16. J. Vainio, I. Vilja, $f(R)$ gravity constraints from gravitational waves. Gen. Relativ. Gravit. 49(8), 99 (2017). arXiv:1603.09551 [astro-ph.CO]

17. M. Ostrogradsky, Mémoires sur les équations différentielles, relatives au problème des isopérimètre. Mem. Acad. St. Petersbourg 6(4), 385 (1850)

18. R.P. Woodard, Avoiding dark energy with $1 / \mathrm{r}$ modifications of gravity. Lect. Notes Phys. 720, 403 (2007). arXiv:astro-ph/0601672

19. H.A. Buchdahl, Mon. Not. R. Astron. Soc. 150, 1 (1970)

20. A.A. Starobinsky, A new type of isotropic cosmological models without singularity. Phys. Lett. B 91, 99 (1980)

21. A.A. Starobinsky, A new type of isotropic cosmological models without singularity. Phys. Lett. 91B, 99 (1980)

22. A.A. Starobinsky, A new type of isotropic cosmological models without singularity. Adv. Ser. Astrophys. Cosmol. 3, 130 (1987). arXiv:1102.0089 [hep-th]

23. S.M. Carroll, M. Hoffman, M. Trodden, Can the dark energy equation-of-state parameter $\mathrm{w}$ be less than -1 ? Phys. Rev. D 68 , 023509 (2003). arXiv:astro-ph/0301273

24. S.M. Carroll, V. Duvvuri, M. Trodden, M.S. Turner, Is cosmic speed-up due to new gravitational physics? Phys. Rev. D 70, 043528 (2004). arXiv:astro-ph/0306438

25. S. Capozziello, Curvature quintessence. Int. J. Mod. Phys. D 11, 483 (2002). arXiv:gr-qc/0201033

26. S. Capozziello, V.F. Cardone, S. Carloni, A. Troisi, Curvature quintessence matched with observational data. Int. J. Mod. Phys. D 12, 1969 (2003). arXiv:astro-ph/0307018

27. M.E. Soussa, R.P. Woodard, The force of gravity from a Lagrangian containing inverse powers of the Ricci scalar. Gen. Relativ. Gravit. 36, 855 (2004). arXiv:astro-ph/0308114

28. V. Faraoni, S. Nadeau, The Stability of modified gravity models. Phys. Rev. D 72, 124005 (2005). arXiv:gr-qc/0511094

29. T. Chiba, 1/R gravity and scalar-tensor gravity. Phys. Lett. B $\mathbf{5 7 5}$, 1 (2003). arXiv:astro-ph/0307338

30. C. Corda, Primordial production of massive relic gravitational waves from a weak modification of General Relativity. Astropart. Phys. 30, 209 (2008). arXiv:0812.0483 [gr-qc]

31. C. Corda, Massive relic gravitational waves from $f(R)$ theories of gravity: production and potential detection. Eur. Phys. J. C 65, 257 (2010). arXiv:1007.4077 [gr-qc]

32. K. Bamba, S.D. Odintsov, Inflation and late-time cosmic acceleration in non-minimal Maxwell- $F(R)$ gravity and the generation of large-scale magnetic fields. JCAP 0804, 024 (2008). arXiv:0801.0954 [astro-ph]

33. S. Nojiri, S.D. Odintsov, Modified $\mathrm{f}(\mathrm{R})$ gravity consistent with realistic cosmology: from matter dominated epoch to dark energy universe. Phys. Rev. D 74, 086005 (2006). arXiv:hep-th/0608008

34. S. Nojiri, S.D. Odintsov, Modified gravity and its reconstruction from the universe expansion history. J. Phys. Conf. Ser. 66, 012005 (2007). arXiv:hep-th/061107

35. S. Nojiri, S.D. Odintsov, The future evolution and finite-time singularities in $\mathrm{F}(\mathrm{R})$-gravity unifying the inflation and cosmic acceleration. Phys. Rev. D 78, 046006 (2008). arXiv:0804.3519 [hep-th]

36. T. Kobayashi, Ki Maeda, Can higher curvature corrections cure the singularity problem in $\mathrm{f}(\mathrm{R})$ gravity? Phys. Rev. D 79, 024009 (2009). arXiv:0810.5664 [astro-ph]

37. V. Faraoni, Modified gravity and the stability of de Sitter space. Phys. Rev. D 72, 061501 (2005). arXiv:gr-qc/0509008

38. S. Capozziello, S. Nojiri, S.D. Odintsov, A. Troisi, Cosmological viability of $f(R)$-gravity as an ideal fluid and its compatibility with a matter dominated phase. Phys. Lett. B 639, 135 (2006). arXiv:astro-ph/0604431 
39. L. Amendola, R. Gannouji, D. Polarski, S. Tsujikawa, Conditions for the cosmological viability of $f(R)$ dark energy models. Phys. Rev. D 75, 083504 (2007). arXiv:gr-qc/0612180

40. M. Akbar, R.G. Cai, Thermodynamic behavior of field equations for $f(R)$ gravity. Phys. Lett. B 648, 243 (2007). arXiv:gr-qc/0612089

41. T. Multamaki, I. Vilja, Spherically symmetric solutions of modified field equations in $\mathrm{f}(\mathrm{R})$ theories of gravity. Phys. Rev. D 74, 064022 (2006). arXiv:astro-ph/0606373

42. T. Multamaki, I. Vilja, Static spherically symmetric perfect fluid solutions in $\mathrm{f}(\mathrm{R})$ theories of gravity. Phys. Rev. D 76, 064021 (2007). arXiv:astro-ph/0612775

43. A. de la Cruz-Dombriz, A. Dobado, A.L. Maroto, Black Holes in f(R) theorie. Phys. Rev. D 80, 124011 (2009). Erratum: [Phys. Rev. D 83, 029903 (2011)]. arXiv:0907.3872 [gr-qc]

44. S.H. Hendi, B. Eslam Panah, S.M. Mousavi, Some exact solutions of $F(R)$ gravity with charged (a)dS black hole interpretation. Gen. Relativ. Gravit. 44, 835 (2012). arXiv:1102.0089 [hep-th]

45. L. Sebastiani, S. Zerbini, Static spherically symmetric solutions in F(R) gravity. Eur. Phys. J. C 71, 1591 (2011). arXiv:1012.5230 [gr-qc]

46. S.H. Hendi, D. Momeni, Black hole solutions in $F(R)$ gravity with conformal anomaly. Eur. Phys. J. C 71, 1823 (2011). arXiv:1201.0061 [gr-qc]

47. S. Asgari, R. Saffari, Vacuum solution of a linear red-shift based correction in $f(R)$ gravity. Gen. Relativ. Gravit. 44, 737 (2012). arXiv: 1104.5108 [gr-qc]

48. S.G. Ghosh, S.D. Maharaj, U. Papnoi, Radiating Kerr-Newman black hole in $f(R)$ gravity. Eur. Phys. J. C 73(6), 2473 (2013). arXiv:1208.3028 [gr-qc]

49. S.H. Hendi, B. Eslam Panah, R. Saffari, Exact solutions of threedimensional black holes: Einstein gravity versus $F(R)$ gravity. Int. J. Mod. Phys. D 23(11), 1450088 (2014). arXiv:1408.5570 [hep-th]

50. Z.Y. Tang, B. Wang, E. Papantonopoulos, Exact charged black hole solutions in D-dimensions in $\mathrm{f}(\mathrm{R})$ gravity. arXiv:1911.06988 [grqc]

51. T. Regge, J.A. Wheeler, Stability of a Schwarzschild singularity. Phys. Rev. 108, 1063 (1957)

52. F.J. Zerilli, Gravitational field of a particle falling in a Schwarzschild geometry analyzed in tensor harmonics. Phys. Rev. D 2, 2141 (1970)

53. K.D. Kokkotas, B.G. Schmidt, Quasinormal modes of stars and black holes. Living Rev. Relativ. 2, 2 (1999). arXiv:gr-qc/9909058

54. H.P. Nollert, Topical review: quasinormal modes: the characteristic 'sound' of black holes and neutron stars. Class. Quantum Gravity 16, R159 (1999)

55. R.A. Konoplya, A. Zhidenko, Quasinormal modes of black holes: from astrophysics to string theory. Rev. Mod. Phys. 83, 793 (2011). arXiv:1102.4014 [gr-qc]

56. E. Berti, V. Cardoso, A.O. Starinets, Quasinormal modes of black holes and black branes. Class. Quantum Gravity 26, 163001 (2009). arXiv:0905.2975 [gr-qc]

57. S.I. Finazzo, R. Rougemont, M. Zaniboni, R. Critelli, J. Noronha, Critical behavior of non-hydrodynamic quasinormal modes in a strongly coupled plasma. JHEP 1701, 137 (2017). arXiv: 1610.01519 [hep-th]

58. P.A. González, E. Papantonopoulos, J. Saavedra, Y. Vásquez, Superradiant instability of near extremal and extremal fourdimensional charged hairy black hole in anti-de Sitter spacetime. Phys. Rev. D 95(6), 064046 (2017). arXiv:1702.00439 [gr-qc]

59. E. Abdalla, B. Cuadros-Melgar, R. Fontana, J. de Oliveira, E. Papantonopoulos, A. Pavan, Instability of a Reissner-NordströmAdS black hole under perturbations of a scalar field coupled to the Einstein tensor. Phys. Rev. D 99(10), 104065 (2019). arXiv: 1903.10850 [gr-qc]
60. P.A. Gonzalez, Y. Vasquez, R.N. Villalobos, Perturbative and nonperturbative fermionic quasinormal modes of Einstein-GaussBonnet-AdS black holes. Phys. Rev. D 98(6), 064030 (2018). arXiv: 1807.11827 [gr-qc]

61. R. Bécar, P.A. González, E. Papantonopoulos, Y. Vásquez, Quasinormal modes of three-dimensional rotating Hořava AdS black hole and the approach to thermal equilibrium. Eur. Phys. J. C 80(7), 600 (2020). https://doi.org/10.1140/epjc/ s10052-020-8169-2. arXiv:1906.06654 [gr-qc]

62. A. Aragón, R. Bécar, P.A. González, Y. Vásquez, Perturbative and nonperturbative quasinormal modes of 4D Einstein-Gauss-Bonnet black holes. Eur. Phys. J. C 80(8), 773 (2020). arXiv:2004.05632 [gr-qc]

63. R.A. Konoplya, A.V. Zhidenko, Decay of massive scalar field in a Schwarzschild background. Phys. Lett. B 609, 377 (2005). arXiv:gr-qc/0411059

64. R.A. Konoplya, A. Zhidenko, Stability and quasinormal modes of the massive scalar field around Kerr black holes. Phys. Rev. D 73, 124040 (2006). arXiv:gr-qc/0605013

65. S.R. Dolan, Instability of the massive Klein-Gordon field on the Kerr spacetime. Phys. Rev. D 76, 084001 (2007). arXiv:0705.2880 [gr-qc]

66. O.J. Tattersall, P.G. Ferreira, Quasinormal modes of black holes in Horndeski gravity. Phys. Rev. D 97(10), 104047 (2018). arXiv: 1804.08950 [gr-qc]

67. M. Lagos, P.G. Ferreira, O.J. Tattersall, Anomalous decay rate of quasinormal modes. Phys. Rev. D 101(8), 084018 (2020). arXiv:2002.01897 [gr-qc]

68. A. Aragón, P.A. González, E. Papantonopoulos, Y. Vásquez, Anomalous decay rate of quasinormal modes in SchwarzschilddS and Schwarzschild-AdS black holes. JHEP 2008, 120 (2020). arXiv:2004.09386 [gr-qc]

69. K. Destounis, R.D.B. Fontana, F.C. Mena, Counterexamples to strong cosmic censorship in asymptotically flat black hole spacetimes. arXiv:2006.01152 [gr-qc]

70. R.D.B. Fontana, P.A. González, E. Papantonopoulos, Y. Vásquez, Anomalous decay rate of quasinormal modes in ReissnerNordström black holes. Phys. Rev. D 103(6), 064005 (2021). arXiv:2011.10620 [gr-qc]

71. A. Aragón, R. Bécar, P.A. González, Y. Vásquez, Massive Dirac quasinormal modes in Schwarzschild-de Sitter black holes: anomalous decay rate and fine structure. Phys. Rev. D 103(6), 064006 (2021). arXiv:2009.09436 [gr-qc]

72. D. Psaltis, D. Perrodin, K.R. Dienes, I. Mocioiu, Kerr black holes are not unique to general relativity. Phys. Rev. Lett. 100, 091101 (2008). arXiv:0710.4564 [astro-ph]

73. G.J. Olmo, The gravity Lagrangian according to solar system experiments. Phys. Rev. Lett. 95, 261102 (2005). arXiv:gr-qc/0505101

74. O.J. Kwon, Y.D. Kim, Y.S. Myung, B.H. Cho, Y.J. Park, Stability of the Schwarzschild black hole in Brans-Dicke theory. Phys. Rev. D 34, 333-342 (1986)

75. P. Cañate, L.G. Jaime, M. Salgado, Spherically symmetric black holes in $f(R)$ gravity: is geometric scalar hair supported ? Class. Quantum Gravity 33(15), 155005 (2016). arXiv:1509.01664 [gr$\mathrm{qc}]$

76. P. Cañate, A no-hair theorem for black holes in $f(R)$ gravity. Class. Quantum Gravity 35(2), 025018 (2018)

77. J. Sultana, D. Kazanas, A no-hair theorem for spherically symmetric black holes in $R^{2}$ gravity. Gen. Relativ. Gravit. 50(11), 137 (2018). arXiv:1810.02915 [gr-qc]

78. T. Karakasis, E. Papantonopoulos, Z.Y. Tang, B. Wang, Black holes of $(2+1)$-dimensional $f(R)$ gravity coupled to a scalar field. arXiv:2101.06410 [gr-qc]

79. Z.Y. Tang, B. Wang, T. Karakasis, E. Papantonopoulos, Curvature scalarization of black holes in $\mathrm{f}(\mathrm{R})$ gravity. arXiv:2008.13318 [grqc] 
80. R. Saffari, S. Rahvar, $f(R)$ gravity: from the pioneer anomaly to the cosmic acceleration. Phys. Rev. D 77, 104028 (2008). arXiv:0708.1482 [astro-ph]

81. S.G. Turyshev, V.T. Toth, G. Kinsella, S.C. Lee, S.M. Lok, J. Ellis, Support for the thermal origin of the Pioneer anomaly. Phys. Rev. Lett. 108, 241101 (2012). arXiv:1204.2507 [gr-qc]

82. J.P. Boyd, Chebyshev and Fourier Spectral Methods. Dover Books on Mathematics, 2nd ed. (Dover Publications, Mineola, 2001)

83. A. Zhidenko, Quasinormal modes of Schwarzschild de Sitter black holes. Class. Quantum Gravity 21, 273 (2004)

84. R.A. Konoplya, A. Zhidenko, High overtones of Schwarzschild-de Sitter quasinormal spectrum. JHEP 0406, 037 (2004)

85. A. Jansen, Overdamped modes in Schwarzschild-de Sitter and a Mathematica package for the numerical computation of quasinormal modes. Eur. Phys. J. Plus 132(12), 546 (2017)

86. V. Cardoso, J.L. Costa, K. Destounis, P. Hintz, A. Jansen, Quasinormal modes and strong cosmic censorship. Phys. Rev. Lett. 120(3), 031103 (2018). arXiv:1711.10502 [gr-qc]

87. K. Destounis, Charged fermions and strong cosmic censorship. Phys. Lett. B 795, 211-219 (2019). arXiv:1811.10629 [gr-qc]

88. B. Mashhoon, Quasi-normal modes of a black hole. Third Marcel Grossmann Meeting on General Relativity (1983)

89. B.F. Schutz, C.M. Will, Black hole normal modes: a semianalytic approach. Astrophys. J. Lett. 291, L33 (1985)

90. S. Iyer, C.M. Will, Black hole normal modes: a WKB approach. 1. Foundations and application of a higher order WKB analysis of potential barrier scattering. Phys. Rev. D 35, 3621 (1987)
91. R.A. Konoplya, Quasinormal behavior of the d-dimensional Schwarzschild black hole and higher order WKB approach. Phys. Rev. D 68, 024018 (2003). arXiv:gr-qc/0303052

92. J. Matyjasek, M. Opala, Quasinormal modes of black holes. The improved semianalytic approach. Phys. Rev. D 96(2), 024011 (2017). arXiv:1704.00361 [gr-qc]

93. R.A. Konoplya, A. Zhidenko, A.F. Zinhailo, Higher order WKB formula for quasinormal modes and grey-body factors: recipes for quick and accurate calculations. Class. Quantum Gravity 36, 155002 (2019). arXiv:1904.10333 [gr-qc]

94. Y. Hatsuda, Quasinormal modes of black holes and Borel summation. Phys. Rev. D 101(2), 024008 (2020). arXiv:1906.07232 [gr-qc]

95. D.P. Du, B. Wang, R.K. Su, Quasinormal modes in pure de Sitter space-times. Phys. Rev. D 70, 064024 (2004). arXiv:hep-th/0404047

96. A. Lopez-Ortega, Quasinormal modes of D-dimensional de Sitter spacetime. Gen. Relativ. Gravit. 38, 1565-1591 (2006). arXiv:gr-qc/0605027 